\title{
Development and utility of an in vitro, fluorescence-based assay for the discovery of novel compounds against dengue 2 viral protease
}

\author{
Gianne Eduard L. Ulanday ${ }^{1,2}$, Kenta Okamoto ${ }^{1}$ and Kouichi Morita ${ }^{1,2,3^{*}}$
}

\begin{abstract}
Background: Dengue disease is one of the most significant vector-borne illnesses in the world. The emergence and re-emergence of dengue infections in many parts of the world affect millions annually and continue to burden public health systems especially in low-income populations. Advances in dengue vaccine development showed promising results; however, protection seems to be suboptimal. There is no licensed chemotherapeutic agent against dengue to date. An ideal scenario of combinatorial vaccination of high-risk individuals and chemotherapy of the diseased during outbreaks may compensate for the meager protection offered by the vaccine. The dengue virus protease is important to viral replication and, as such, has been identified as a potential target for antivirals. It is, therefore, our objective to establish and optimize an appropriate screening method for use during the early stages of drug development for dengue.
\end{abstract}

Methods: In this study, we developed and optimized a biochemical assay system for use in screening compound libraries against dengue virus protease. We tested the selected protease inhibitors with a cell-based assay to determine inhibition of viral replication.

Results: We have presented direct plots of substrate kinetics data showing an apparent inhibition of the protease at excessive substrate concentrations. The most common sources of interference that may have affected the said observation were elucidated. Finally, a screen was done on an existing compound library using the developed method. The compounds selected in this study showed inhibitory activity against both the recombinant dengue protease and cell-based infectivity assays.

Conclusions: Our study shows the practicality of a customized biochemical assay to find possible inhibitors of dengue viral protease during the initial stages of drug discovery.

Keywords: Dengue virus, Antiviral screening, Dengue protease, NS2B-NS3pro

\section{Background}

Dengue disease is a public health problem that continues to affect approximately 100 countries globally with an estimated 390 million cases per year of which majority are children. The burden of disease lies mostly in the Asia-Pacific yet the number of affected countries

\footnotetext{
* Correspondence: moritak@nagasaki-u.ac.jp

${ }^{1}$ Department of Virology, Institute of Tropical Medicine, Nagasaki University,

1-12-4 Sakamoto, Nagasaki 852-8523, Japan

${ }^{2}$ Graduate School of Biomedical Sciences, Nagasaki University, Nagasaki,

Japan

Full list of author information is available at the end of the article
}

continues to rise as it emerges into new areas putting more people at risk [1]. Trans-ovarial transmission of dengue virus (DENV) within mosquito populations can facilitate transmission between or within epidemics, thus complicating disease control strategies $[2,3]$. Human migration and vector invasiveness contributed to the emergence and re-emergence of dengue in Europe and Asia thus highlighting the need for a more proactive approach in dealing with this neglected disease $[4,5]$.

The causative agent, dengue virus, is a single-stranded RNA virus belonging to the Flavivirus genus of the 
family Flaviviridae. It has four serotypes wherein each DENV serotype is phylogenetically distinct, suggesting that each could be considered as a separate virus. Transmission is accomplished through the bite of mosquitoes, prominently, Aedes aegypti and Aedes albopictus. In humans, intrinsic incubation period ranges 3-10 days after the bite of infected mosquitoes [6, 7]. Although dengue infection is generally asymptomatic, it may result in a wide spectrum of clinical disease, ranging from a mild flu-like syndrome (dengue fever) to the most severe forms of the disease, which are characterized by coagulopathy, increased vascular fragility, and permeability (dengue hemorrhagic fever). The latter may progress to hypovolemic shock (dengue shock syndrome). Poor clinical outcomes have been linked to secondary infections [8]. However, not all heterologous secondary infections cause subsequent severe forms of the disease. Host and viral factors, age, and timing of infection are some of the aspects to be considered [9].

The lack of chemotherapeutic agents and heavy reliance on vector control complicate the management and prevention of dengue. Although recent advances in vaccine development have proven that a tetravalent preparation is possible, field trials showed only around $50 \%$ vaccine efficacy and meager protection against specific serotypes $[10,11]$. One study estimates vaccine protection in a population from 24 to $54 \%$ according to their models [12]. This observation led to more questions regarding the mechanisms involving immunity against dengue and proxy determinants of protection. The success of marketed drugs targeting viral enzymes such as in hepatitis $\mathrm{C}$ virus (HCV) and human immunodeficiency virus (HIV) has directed the interest of researchers towards flaviviral proteases. Recent literature suggests that the viral proteins of DENV will probably have a lower tendency to experience resistance-causing mutations than in the case of $\mathrm{HCV}$ and therefore is suitable as potential drug targets [13].

The 11 kilobase DENV genome encodes a polyprotein containing the structural and nonstructural (NS) proteins. The polyprotein is processed by several proteases, including that of the host. Similar with other flaviviruses, the dengue virus protease is considered as a trypsin-like serine protease located within NS3 [13]. The NS3 has two enzymatic domains, a serine protease (NS3pro) and helicase component located at the amino and carboxyl terminals, respectively. Interestingly, DENV protease has a marked cleavage site preference for dibasic residues which is distinct from native trypsin [14]. The protease domain requires the 40-amino acid central hydrophilic region of NS2B $(\mathrm{NS} 2 \mathrm{~B}(\mathrm{H}))$ as a cofactor which forms as part of the substrate recognition region [13]. The NS2B $(\mathrm{H})$ NS3 protease complex (NS2B(H)-NS3pro) is vital for the post-translational processing of the polyprotein precursor and is essential for viral replication and maturation of infectious virions [14]. Considering these points, the dengue protease component located within NS3 is a very promising target for antiviral development. Hence, the present study aims to establish and optimize an assay for use during the early stages of drug development for dengue.

In this study, we have constructed and expressed a recombinant DENV2 viral protease for use in screening of potential lead compounds. Our optimized biochemical assay provided a straightforward and practical alternative to currently available assay methodologies. Also, we have shown and compared two enzyme kinetic models to guide future researchers in analyzing their data. The established assay identified potential lead compounds which were confirmed by cell-based assays.

\section{Methods}

\section{Cloning of pET28a/DENV NS2B(H)-G4SG4-NS3pro of DENV2}

The DENV NS2B(H)-NS3pro gene sequence containing hydrophilic residues 49-96 of NS2B and N-terminal 180 amino acids of NS3 were amplified from DENV-2 16681 infectious clone (GenBank Accession: U87411) (given by Richard Kinney, Center for Disease Control and Prevention, Division of Vector-borne Diseases, Fort Collins, CO, USA) analogous as described previously $[15,16]$. Briefly, the primer pairs Nhe1-NS2B-F and (G4SG4)NS2B-R, (G4SG4)-NS3-F, and Xho1-NS3-R were used during polymerase chain reaction (PCR) to produce the fragments NS2B(H)-Linker and Linker-NS3pro, respectively. Subsequently, overlapping PCR was performed through the combination of both templates with primer pairs Nhe1-NS2B-F and Xho1-NS3-R to produce the final full length NS2B(H)-G4SG4-NS3pro incorporating an overlapping region of 27 nucleotides and restriction endonuclease recognition sites at both ends. The PCR product was then digested with NheI and XhoI before cloning into pET28a vector (Novagen) to yield the Nterminal polyhistidine-tagged fusion protein. All PCR reactions were done using Phusion ${ }^{\circ}$ High-Fidelity DNA Polymerase (New England Biolabs). Construct sequence was confirmed by automated Sanger sequencing using BigDye Terminator v3.1 Cycle Sequencing Kit (Applied Biosystems).

\section{Expression and purification of DENV2 NS2B(H)-G4SG4- NS3pro (rNS2B3pro)}

The pET28a construct containing the DENV NS2B $(\mathrm{H})$ G4SG4-NS3pro was transformed into BL21-CodonPlus (DE3)-RIPL strain (Agilent) which provides correction for possible codon usage bias. Cells were then grown in 250$\mathrm{mL}$ Luria-Bertani broth containing $50 \mu \mathrm{g} / \mathrm{mL}$ kanamycin until $\mathrm{OD}_{600}$ reached 0.6-0.8. Protein expression was induced by adding isopropyl $\beta$-D-1-thiogalactopyranoside 
(Wako, Japan) at $0.5 \mathrm{mM}$ final concentration for $3 \mathrm{~h}$ at $37^{\circ} \mathrm{C}$. Cells were then harvested by centrifugation at 7000 $\mathrm{rpm}$ for $10 \mathrm{~min}$ at $4{ }^{\circ} \mathrm{C}$ and the cell pellet was resuspended in 30-mL cold lysis buffer (phosphate-buffered saline) prior to mechanical lysis by sonication on ice. The cell suspension was then centrifuged at 15,000 rpm for $30 \mathrm{~min}$ to separate the supernatant from the insoluble fraction. The collected soluble fraction was then filtered through a $0.45-\mu \mathrm{m}$ polyvinylidene fluoride (PVDF) syringe filter unit (Merck-Millipore).

The filtrate containing the histidine-tagged fusion protein was subsequently purified using gravity flow immobilized metal affinity chromatography (IMAC). Briefly, Cobalt TALON $^{\circ}$ (Takara-Clontech, Japan) was loaded into an open column to approximately $1-\mathrm{mL}$ bed volume. The column was subsequently washed with 20 volumes of distilled water prior to column equilibration with another 20 volumes of lysis buffer. The previously filtered sample was loaded then washed with $40-\mathrm{mL}$ native wash buffer ( $20 \mathrm{mM}$ imidazole in lysis buffer) to remove other contaminating proteins. Elution was started upon addition of $5 \mathrm{~mL}$ each of 100, 200, 400, and $800 \mathrm{mM}$ imidazole (Wako, Japan) suspended in lysis buffer. The elution fractions were then combined in pairs, 100 and $200 \mathrm{mM}, 400$ and $800 \mathrm{mM}$ before concentrating by centrifugation using Amicon Ultra-15 (Merck-Millipore). The fraction was further purified using Superdex 75 (GE Life Sciences, Japan) gel filtration column with running buffer containing $50 \mathrm{mM}$ Tris- $\mathrm{HCl}(\mathrm{pH} 8-9)$ and $100-300 \mathrm{mM} \mathrm{NaCl}$ with a flow rate of $0.4 \mathrm{~mL} / \mathrm{min}$. Detection was done using 280 and $220 \mathrm{~nm}$. The different fractions were visualized by Coomassie-stained sodium dodecyl sulfate polyacrylamide gel electrophoresis (SDS-PAGE) gels and antihistidine Western blots to confirm the presence of the target protein. Protein concentration was determined using Direct Detect ${ }^{\oplus}$ infrared spectrometer (EMD Millipore, USA).

\section{Confirmation of target protein using SDS-PAGE and immunoblotting (Western blot)}

The different elution fractions were collected for the detection of the target protein. Approximately $10 \mu \mathrm{L}$ of each fraction was loaded for SDS-PAGE analysis and immunoblotting. In Western blotting, PVDF membrane containing the transferred protein was blocked by BlockAce solution (Dainippon Sumitomo Pharma, Osaka, Japan) then treated with mouse anti-histidine monoclonal antibody as primary antibody and sequentially stained by horseradish peroxidase (HRP)-conjugated anti-mouse antibody as secondary antibody. The HRP reaction was developed and measured via chemiluminescence using ImageQuant ${ }^{\mathrm{Tm}}$ LAS 4000 (GE Life Sciences, Japan) biomolecular imager.

\section{Validation of protease activity and optimization of assay components}

A standard amount of purified protease was added into wells of a black, flat-bottom 96-well plate containing assay buffer (200 mM Tris- $\mathrm{HCl}, 20 \%$ glycerol, $\mathrm{pH}$ 9.5) then mixed. Reaction was started upon the addition of the substrate and was monitored via ARVO MX 1420 Multilabel Counter microplate reader (Perkin-Elmer, Japan) after $30 \mathrm{~min}$. Assays were done at room temperature with a final volume of $100 \mu \mathrm{L}$.

A substrate assay was done by comparing five different coumarin-based fluorogenic substrates used in other flavivirus protease studies [17-20]. Briefly, a single concentration $(1000 \mu \mathrm{M})$ of the selected substrates was added similarly as described above. Reactions were started with the addition of purified recombinant protease (rNS2B3pro) with a final concentration of $100 \mu \mathrm{g} / \mathrm{mL}$.

It has been mentioned in other studies that glycerol affects the stability of some proteins in suspension; therefore, assay buffer containing various concentrations of glycerol was studied to determine the optimal level to be used in the system [21]. The fluorogenic peptide substrate Bz-nKRR-MCA (Peptides International) was used to monitor enzyme activity.

An inhibitor control is needed to validate screening; therefore, different concentrations of a protease inhibitor, aprotinin, were added into wells containing $100 \mu \mathrm{g} /$ $\mathrm{mL}$ rNS2B3pro and mixed prior to addition of the substrate, Bz-nKRR-MCA. Fluorescence emission was monitored every $5 \mathrm{~min}$ for $1 \mathrm{~h}$ to determine its stability under assay condition.

Substrate kinetics experiments were done using various concentrations of Bz-nKRR-MCA $(10-1000 \mu \mathrm{M})$ which were mixed in wells containing an assay buffer. Reaction was initiated upon the addition of purified rNS2B3pro to a final concentration of $1 \mu \mathrm{g} / \mathrm{mL}$. Substrate hydrolysis was monitored for $30 \mathrm{~min}$ using ARVO MX 1420 Multilabel Counter microplate reader (PerkinElmer, Japan) at room temperature. To estimate substrate kinetic values, the rates of reaction were plotted as a function of substrate concentration and fitted into an appropriate model.

\section{Cell-based assays \\ Cell viability assay}

Cell viability was determined using CellTiter $96^{\circ}$ NonRadioactive Cell Proliferation Assay (MTT) (Cat \#G4000, Promega) according to the manufacturer's recommendations. Briefly, compounds were resuspended into appropriate concentrations using culture media (Minimum Essential Medium with $2 \%$ fetal calf serum) prior to addition into wells containing Vero cells in confluence. Formazan formation was detected according to protocol after $72 \mathrm{~h}$ of incubation at $37{ }^{\circ} \mathrm{C}$ with $5 \% \mathrm{CO}_{2}$. Two 
duplicate sample measurements were compared with the vehicle control (1\% dimethyl sulfoxide (DMSO) in culture media) to determine the compound maximum nontoxic dose (MNTD).

\section{Cell-based infectivity assays}

Focus formation assays were done to determine the effect of the compound on viral infectivity. Cells were infected in close analogy to the procedure mentioned previously except that Vero cells were used instead [22]. Serial dilutions of the compound in culture medium were mixed with dengue virus 2 isolated from patient serum, 00-St-022 (GenBank Accession: KF744401.1) then added into wells containing Vero cells. The plates were then incubated at $37{ }^{\circ} \mathrm{C}$ with $5 \% \mathrm{CO}_{2}$ for $72 \mathrm{~h}$ prior to detection of intracellular DENV antigens.

\section{Antigen detection enzyme-linked immunosorbent assay (ELISA)}

ELISA was performed to quantitatively determine the level of viral antigen secreted in culture fluid posttreatment as previously described [22, 23]. Briefly, 96well microplates were coated with anti-flavivirus IgG from purified pooled serum and then blocked with BlockAce (Dainippon Sumitomo Pharma, Osaka, Japan) in PBS. The 5-day post-infection culture fluids were added, the plates washed, and HRP-conjugated mAb 12D11/7E8 was mixed into wells. The HRP reaction was detected by adding o-phenylenediamine dihydrochloride (OPD) substrate in the presence of $0.02 \%$ hydrogen peroxide, for $30 \mathrm{~min}$ at room temperature, and stopped with $1 \mathrm{~N}$ sulfuric acid prior to OD measurement at $492 \mathrm{~nm}$.

\section{Statistical analysis}

Analyses were done using GraphPad Prism version 6.07 for Windows, GraphPad Software, La Jolla, California, USA, www.graphpad.com. Estimation of parameters including the $50 \%$ inhibitory concentration $\left(\mathrm{IC}_{50}\right)$ were computed using the above-mentioned software with terms comparable to the recommendations of the International Union of Pharmacology Committee on Receptor Nomenclature and Drug Classification [24, 25]. Statistical treatment of data is specified under each analyses with significance assigned at $p<0.05$. All experiments were done in duplicates at least twice unless stated otherwise.

\section{Results}

Cloning, expression, and purification of recombinant DENV2 NS2B3pro

The DENV2 NS2B(H)-G4SG4-NS3pro plasmid construct included the hydrophilic residues 49-96 of NS2B and N-terminal 180 amino acids of NS3 joined by a glycine linker (Fig. 1a) [26]. The construct sequence was verified by automated sequencing (Sanger method) prior to transformation into expression cells. The $28-\mathrm{kDa}$ recombinant protease (rNS2B3pro) was purified using cobalt immobilized metal affinity chromatography. Different elution fractions were collected and visualized by Coomassie Brilliant Blue staining and anti-histidine immunoblotting. Anomalous migration patterns on SDSPAGE were observed as in the previous studies (Fig. 1b) $[18,27,28]$. The $100 \mathrm{mM}$ imidazole eluted fraction was further concentrated into approximately $1 \mathrm{~mL}$ prior to size exclusion chromatography. Further purification was attempted using Superdex 75 (GE Life Sciences, Japan) gel filtration column (Additional file 1: Figure S1). Since the HPLC result was comparable to the purity produced by the crude open column IMAC, the latter method was then utilized for the remainder of the study.

\section{Development of the assay system}

Assay conditions mentioned in literature were optimized with emphasis on substrate and control inhibitor selection and additive concentration. Based on the peptide sequence suggested by Li et al. as control, Additional file 1: Figure S2A shows the comparison of different flaviviral fluorogenic substrates at a fixed enzyme concentration as used in other protease studies [26, 27, 29, 30]. The peptide substrate with a sequence norleucinelysine-arginine-arginine (nKRR) was confirmed to have a higher substrate cleavage activity than that produced by substrates used in other dengue, West Nile, and Japanese encephalitis virus protease assays. The difference in activity produced between the protective groups, benzoyl $(\mathrm{Bz})$ and acetyl $(\mathrm{Ac})$, also seems to be insignificant.

Different concentrations of glycerol were assayed wherein $20 \%$ was considered to be standard in other studies [29, 31, 32]. Truly, the difference in activity among the 0-60\% concentrations can be observed (Additional file 1: Figure S2B) upon comparison against $20 \%$ glycerol.

Assumption of a blank (no substrate) as $100 \%$ inhibition in a compound screen is suboptimal; therefore, a control inhibitor is necessary for screening. Literature search led to the selection of aprotinin for further experiments [29]. Different concentrations of aprotinin were mixed with the recombinant protease prior to fluorescence monitoring every 5 min for $1 \mathrm{~h}$. Additional file 1 : Figure S3A shows that at $30 \mathrm{~min}$, a minimum of $40 \mu \mathrm{M}$ aprotinin was able to inhibit protease activity comparable to that of buffer only controls. The same concentration was able to maintain inhibition up to $1 \mathrm{~h}$ (Additional file 1: Figure S3B).

The Michaelis-Menten model requires certain assumptions to be fulfilled for the accurate estimation of kinetic parameters. Firstly, the reaction should be observed at initial velocity conditions, wherein the amount 


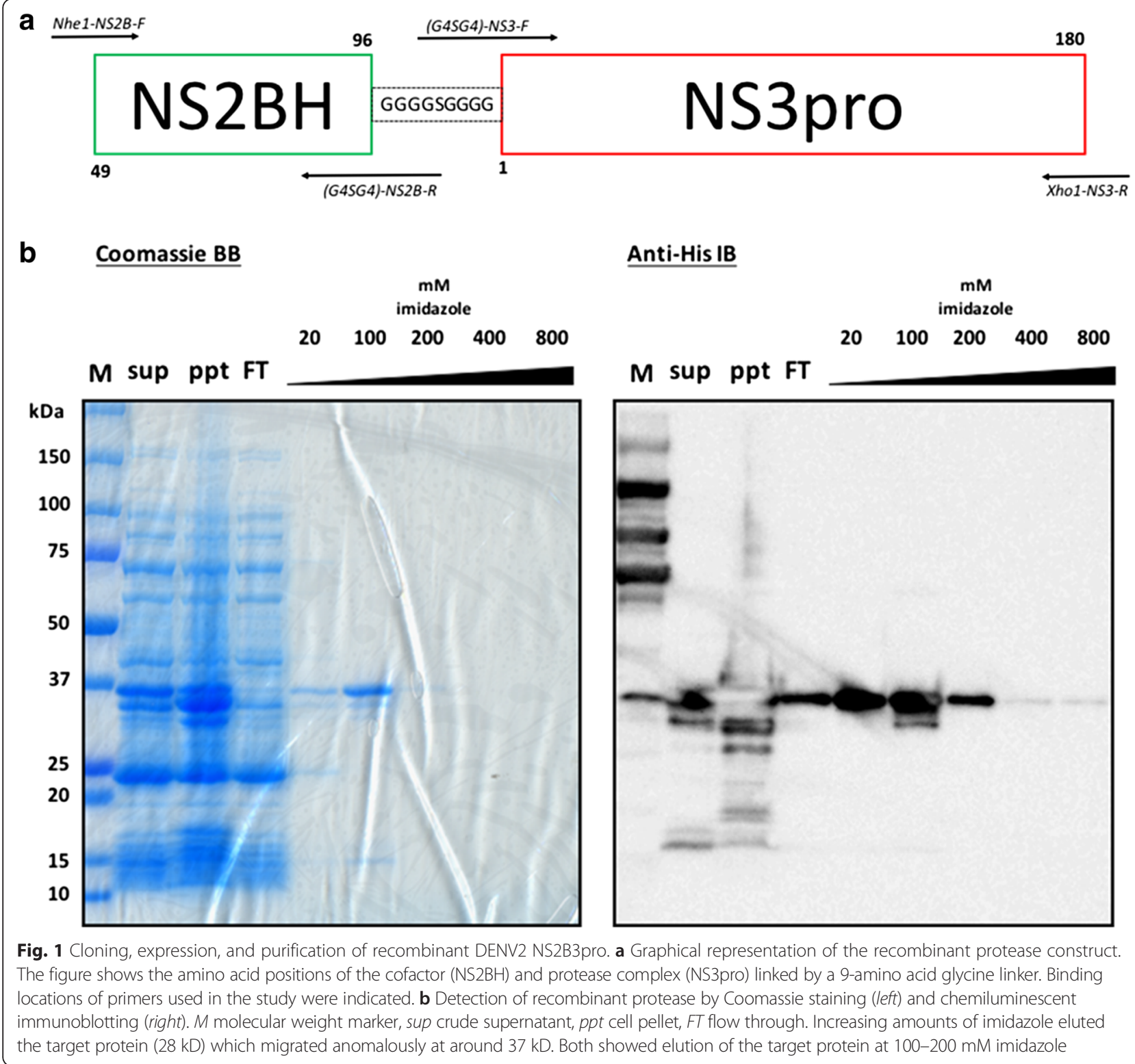

of product formed is negligible to be considered for reversion into the substrate. Figure 2 shows the kinetics of the recombinant protease produced with increasing concentration of the fluorogenic substrate Bz-nKRR-MCA. The protease seems to follow the ubiquitous MichaelisMenten model kinetics up to around $500 \mu \mathrm{M}$ of the substrate. However, further increase in the concentration of the substrate led to a model similar to that of substrate inhibition. To further validate the observation, elimination of other extraneous factors such as photodetector saturation, fluorescence quenching, or inner filter effect was necessary. Initially, a fluorophore standard assay was done wherein free 7-amino-4-methylcoumarin (AMC) (Sigma-Aldrich) was serially diluted, processed, and read as mentioned above with the exception of enzyme addition. Photodetector saturation was observed on concentrations at least ten times the maximum fluorescence emitted by substrate cleavage experiments (data not shown). Consequently, the free AMC concentration producing half the maximal values on the standard AMC assay was chosen for the validation assay (Fig. 3a). To determine whether significant absorption of fluorescence emitted by the liberated fluorophore occurred with increasing concentrations of the substrate, simulation of fluorophore release upon hydrolysis was done using free AMC $(\sim 30 \mu \mathrm{M})$ added into wells containing the uncleaved peptide substrate. Figure $3 \mathrm{~b}$ shows that there is no significant difference in the levels of fluorescence detected among the groups suggesting that fluorophore quenching by the peptide substrate is highly unlikely. Michaelis 


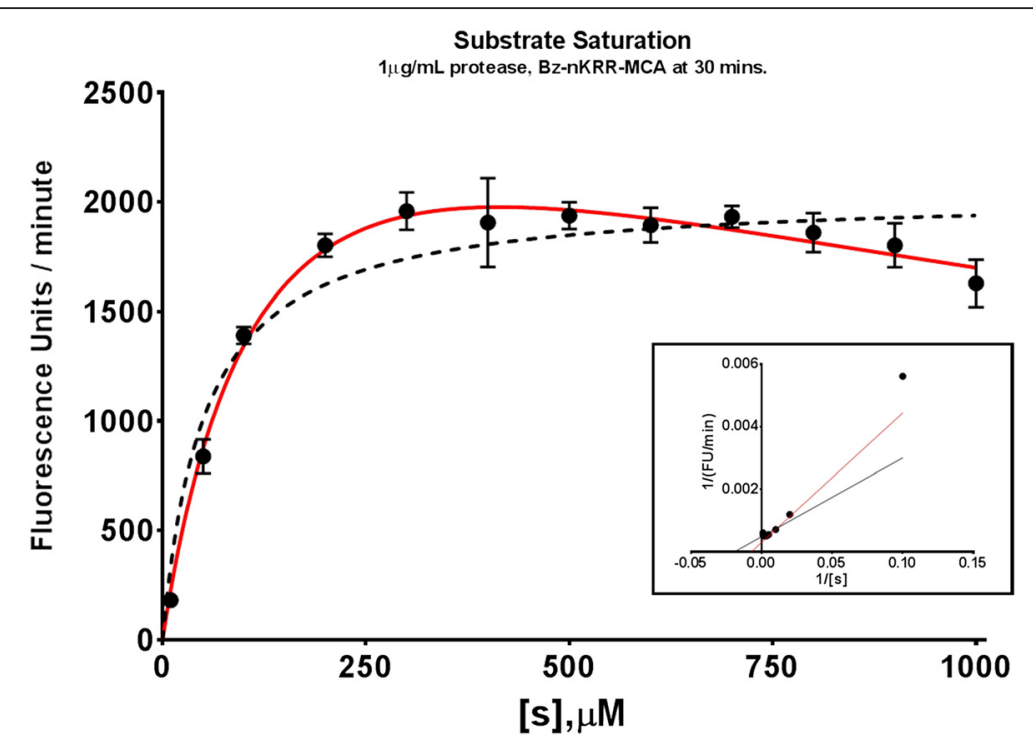

Fig. 2 Substrate saturation assay. Fluorogenic substrate was serially diluted prior to addition of $1 \mu \mathrm{g} / \mathrm{mL}$ purified recombinant DENV2 NS2B3pro. Baseline values at time 0 were subtracted. Two nonlinear regression models of enzyme kinetics were plotted; Michaelis-Menten model (black dashed line) and substrate inhibition model (red line). Inset shows Lineweaver-Burk plot of the same. Error bars indicate standard deviation (SD)

constant estimation based on the different models used is presented under Table 1. Results suggest that the model accounting for substrate inhibition fits the data points regardless of maximal substrate concentration.

\section{Selection of compounds}

The optimized protease screening system based on results mentioned was employed as a duplicate, single concentration $(50 \mu \mathrm{g} / \mathrm{mL}$ compound, $1 \mu \mathrm{g} / \mathrm{mL}$ purified protease) assay. Inhibition of protease activity was monitored with fluorogenic peptide substrate, $100 \mu \mathrm{M}$ Bz-nKRR-MCA, at 30 min using ARVO MX 1420 Multilabel Counter microplate reader (Perkin-Elmer, Japan). Compounds inhibiting at least $85 \%$ of the enzyme control reaction were selected for further investigation. Approximately 500 compounds were screened and three were chosen for further study. Protease $\mathrm{IC}_{50}$ values of the three selected compounds ranged from 5 to $14 \mu \mathrm{g} / \mathrm{mL}$ based on the conditions set above (Table 2). Dose response curves plotting concentrations from $50 \mu \mathrm{g} / \mathrm{mL}$ is shown in Fig. 4.

\section{Cell viability assay}

The three compounds were diluted with culture media and added into wells containing Vero cells in confluence prior to detection of formazan formation $72 \mathrm{~h}$ post treatment. The results showed cytotoxicity of all compounds at $30 \mu \mathrm{g} / \mathrm{mL}$. However, there was no significant difference in the cell viability versus $1 \%$ DMSO control with $10 \mu \mathrm{g} / \mathrm{mL}$ except for ASDN-10343 which showed marginal effect (Fig. 5a). Hence, further experiments were done at concentrations starting $10 \mu \mathrm{g} / \mathrm{mL}$ which was considered to be the maximum nontoxic dose (MNTD).

\section{Cell-based infectivity assays}

The effect of the selected compounds on viral infectivity was assessed via focus formation assay. Figure $5 b$ demonstrates the reduction in focus number by the compounds compared to vehicle control (1\% DMSO in culture media). Although the strength of inhibition varied by compound, at least $50 \%$ inhibition at $5 \mu \mathrm{g} / \mathrm{mL}$ minimum concentration was seen among the selected. Significant inhibition was observed at all concentrations tested for ASDN-10321 and ASDN-10343. Qualitative inspection by microscopy of cell growth during the experiments showed congruence with the previous cell viability results (data not shown).

\section{Antigen detection enzyme-linked immunosorbent assay (ELISA)}

The secretion of viral antigens into the culture fluid was also assessed by employing ELISA. Surprisingly, ASDN10321 also demonstrated significant inhibition at all concentrations tested (Fig. 5c).

\section{Discussion}

The dengue virus protease is a known attractive target for antiviral development. Inhibitors targeting viral proteases have been gaining popularity after recent advances in drug development for hepatitis $\mathrm{C}$ and human immunodeficiency viruses, to name a few [33].

Although DENV protease assays have been widely mentioned in literature, it is imperative that the assay conditions be checked and specifically fitted to our objectives. In this study, a biochemical assay for screening of potential inhibitors against dengue protease has been established. The developed assay has identified lead 

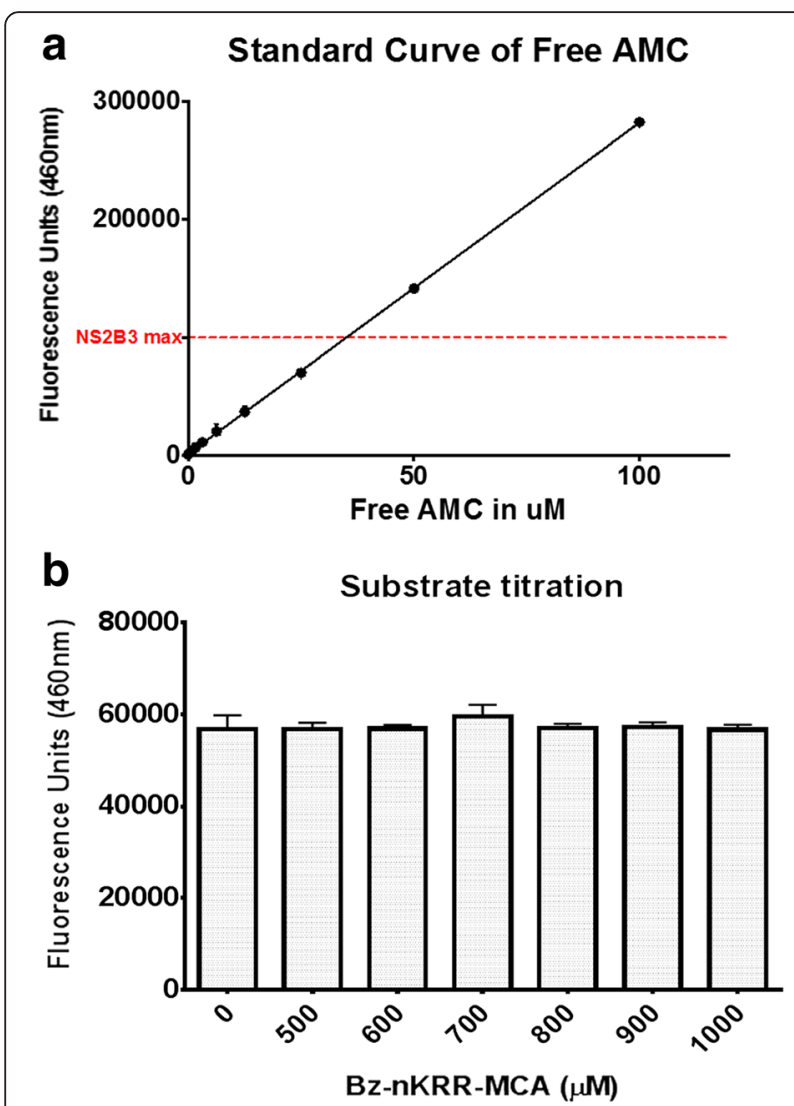

Fig. 3 Free fluorophore assay. a Serially diluted-free fluorophore (AMC) was evaluated in similar conditions as with the protease assay to determine photodetector saturation. The maximal fluorescence value (approx. 150,000 units) elicited during cleavage conditions was indicated with a dashed line. $\mathbf{b}$ Substrate titration assay. Different concentrations of fluorogenic peptide Bz-nKRR-MCA were mixed with $30 \mu \mathrm{M}$ of free $\mathrm{AMC}$ and read after $30 \mathrm{~min}$ of incubation to detect fluorescence quenching. Statistical analysis involved two-way ANOVA with Bonferroni correction. Error bars indicate SD

compounds affecting DENV replication which was later confirmed by cell-based infection assays.

We successfully cloned and expressed DENV2 viral protease in Escherichia coli and were able to produce the final product with adequate purity for use in an assay. Different isolated peaks were seen during size exclusion chromatography; nonetheless, their SDS-PAGE profiles were similar (Additional file 1: Figure S1). A previous study suggested that this was possibly due to products of premature translation termination and/or
Table $\mathbf{2} \mid C_{50}$ values of selected compounds. Best-fit values and their respective $95 \%$ confidence intervals are presented

\begin{tabular}{lll}
\hline & $I C_{50}(\mu \mathrm{g} / \mathrm{mL})$ & $95 \% \mathrm{Cl}(\mu \mathrm{g} / \mathrm{mL})$ \\
\hline ASDN-10287 & 9.786 & 9.015 to 10.62 \\
ASDN-10321 & 5.498 & 5.251 to 5.756 \\
ASDN-10343 & 14.37 & 13.26 to 15.57
\end{tabular}

Determination of was $\mathrm{IC}_{50}$ done using nonlinear regression fitting

existence of aggregated recombinant protease species. Nevertheless, they determined that the different peak fractions did not differ in enzymatic activity [34].

Our study used direct plotting of kinetics data by nonlinear regression wherein estimation is straightforward. It lacks certain disadvantages of linear kinetic plots such as excessive effect of values at the lowest substrate concentrations. The substrate inhibition observed in our study was consistent with the results of Tomlinson et al. using West Nile and dengue viruses [17, 20,35]. Our study used a fluorogenic substrate based on the results of profiling studies conducted by Li et al. which showed over 100-fold improvement in activity compared to other existing sequences, including those used by Tomlinson [26]. Nevertheless, the inhibition was still observed. This may point out that the length of the substrate and the amino acid sequence interacting with the $\mathrm{P} 3 / \mathrm{P} 4$ positions of the enzyme active site may have contributed to this observation. Although the most common sources of possible interference in our observation has been ruled out, other possibilities such as insolubility of end product, intermediary product formation, or $\mathrm{pH}$ changes upon substantial substrate cleavage may lead into inefficient release or detection of the fluorescent analyte. Thus, this observation needs to be looked into more closely.

One of the drawbacks of having a standardized singlepoint screening assay is that there is no account of differences in test compound characteristics. In our study, solubility of some compounds in the aqueous buffer of the protease system was a major difficulty. Visual inspection of the compounds suspended in buffer was necessary to ensure homogeneity during assays. Overtly insoluble compounds were excluded from further analysis. The assay system also suffered problems with autofluorescent compounds which was also a limitation. The drawbacks encountered were not entirely new to drug screening [3638]. In a study by Simeonov et al., approximately $5 \%$ of

Table 1 Michaelis constant $\left(K_{m}\right)$ approximation

\begin{tabular}{llll}
\hline & \multicolumn{1}{c}{ Michaelis-Menten model } & & \multicolumn{2}{c}{ Substrate inhibition model } \\
\cline { 2 - 3 } Max substrate conc. & $1000 \mu \mathrm{M}$ & $500 \mu \mathrm{M}$ & $1000 \mu \mathrm{M}$ \\
\hline$K_{m}$ in $\mu \mathrm{M}(95 \% \mathrm{Cl})$ & 51.23 & 76.24 & 135.0 \\
& $(36.80$ to 65.66) & $(58.24$ to 94.24) & $(99.52$ to 170.4) \\
Adjusted R square & 0.8965 & 0.9638 & 0.9687 \\
\hline
\end{tabular}

Estimates of $K_{m}$ derived from varied nonlinear regression models and substrate maximal concentrations at initial velocity conditions were presented 


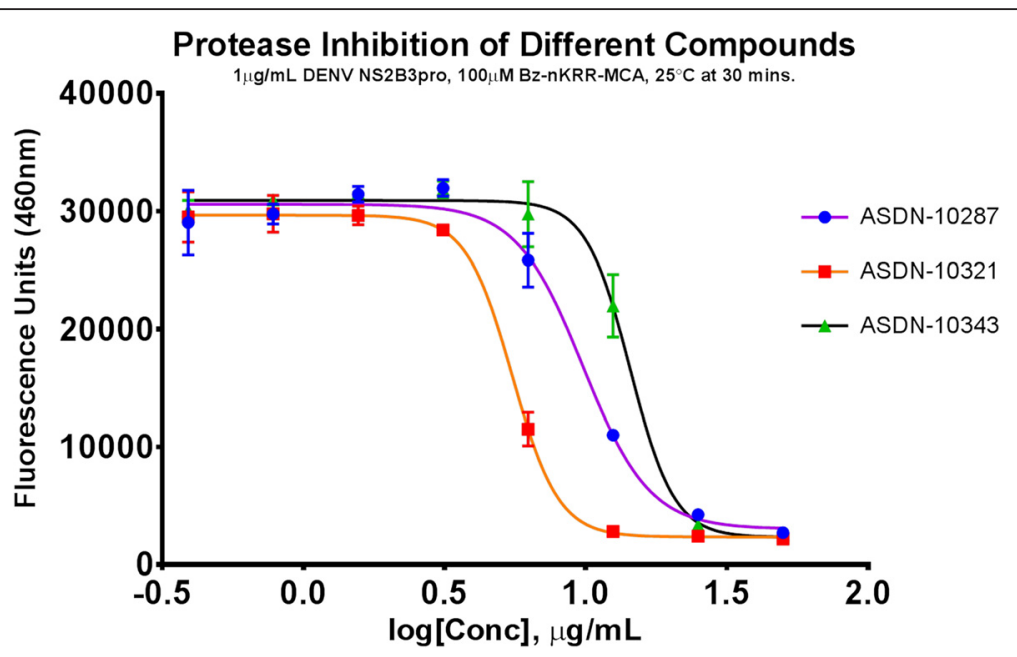

Fig. 4 Protease inhibition profile of selected compounds. Serial dilutions of compounds were mixed with $1 \mu \mathrm{g} / \mathrm{mL}$ purified recombinant DENV2 NS2B3pro prior to addition of $100 \mu \mathrm{M}$ Bz-nKRR-MCA fluorogenic substrate and read after $30 \mathrm{~min}$ at $25^{\circ} \mathrm{C}$. Error bars indicate SD
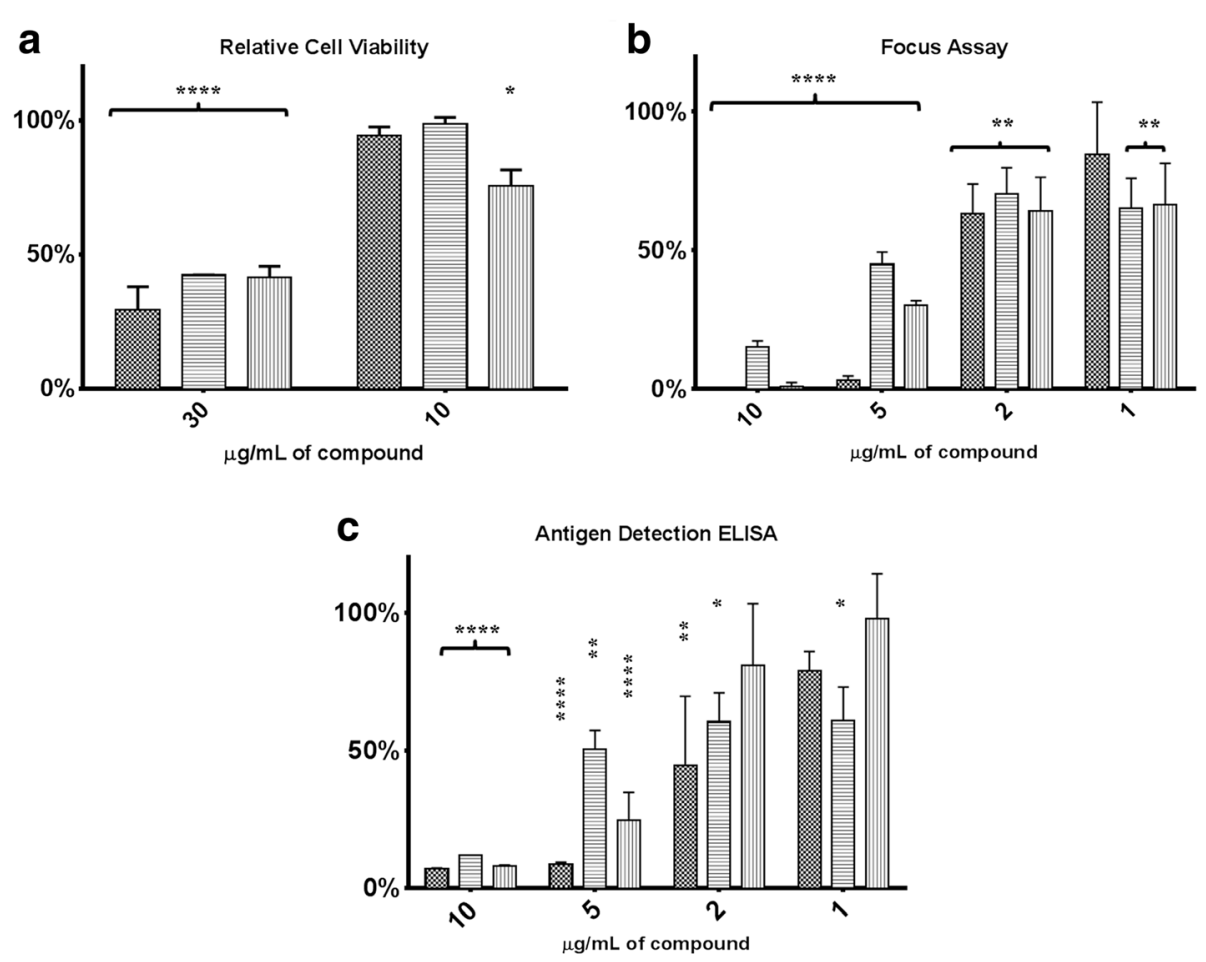

\ASDN-10287 首ASDN-10321 【ASDN-10343

Fig. 5 Cell-based assays. a Cell viability assay. Effect of compounds on cell viability was observed via the MTT assay. Results are expressed as percentage of live cells relative to vehicle (1\% DMSO) control. b Cell-based infectivity assay of compounds. Selected compounds were examined at different concentrations and evaluated for antiviral effect on Vero cells. Activity is reported as percentage relative to infected vehicle-treated only control. c Antigen detection ELISA. Effect of the different compounds on the detected viral antigens in culture fluid was examined. Results are expressed as percentage relative to infected vehicle-treated only control. Statistical analysis involved two-way ANOVA with Bonferroni correction. Error bars indicate SD 
the tested library containing 70,000 compounds were more fluorescent than $10 \mathrm{nM}$ of their probe [39].

Recently, recombinant expression of uncoupled NS2B cofactor and NS3 protease has been successful [40, 41]. In vitro fluorescence-based protease assays showed minimal differences between the characteristics of the unlinked and glycine-linked constructs. The most prominent distinction is the apparent lower substrate affinity of the linked construct using longer peptide substrates. They suggested that the linker may have reduced the flexibility of the protease complex therefore restricting access to the active site [40]. This may explain the apparent inhibition observed in our study wherein the excess substrate may have blocked the entry to the site and that the limited flexibility reduced the ability of the protease to form an "induced fit." Nevertheless, the glycine-linked construct provides an adequate model as target for small, active site inhibitors.

\section{Conclusions}

In this study, we described the successful development of a dengue- 2 protease biochemical assay as a useful tool for screening of potential inhibitory compounds. Followup experiments of selected compounds confirmed the effects against dengue virus 2 replication in cells. The results will serve as basis for further modifications to the lead compound structure so as to increase its activity against dengue virus and improve its selectivity. Preliminary experiments are currently being undertaken to analogs of the lead compounds as part of a structure-activity relationship study. An opportunity to test the same compounds with other flaviviruses is under consideration.

\section{Additional file}

Additional file 1: Table S1. Primers used in the amplification of the target sequence. Lower case letters denote viral sequence; italicized letters denote sequence comprising the glycine linker region. Letters in bold indicate restriction endonuclease recognition sequences. Figure S1. Fraction collections using size exclusion chromatography. (A) Absorbance detected per each fraction as a function of time. (B) Coomassie SDS-PAGE analysis, (C) anti-histidine Western blot. $\mathrm{M}=$ marker, $\mathrm{W}=20 \mathrm{mM}$, $1=100 \mathrm{mM}, 2=200 \mathrm{mM}, 3=400 \mathrm{mM}, 4=800 \mathrm{mM}$ Imidazole. Figure S2A. Comparison of different flaviviral protease substrates. Single concentration of substrate $(1000 \mu \mathrm{M})$ was added into wells containing a fixed amount of DENV2 rNS2B3pro $(100 \mu \mathrm{g} / \mathrm{mL})$ and measured at $30 \mathrm{~min}$. Figure shows relative fluorescence compared to Bz-nKRR-MCA under the same conditions. Error bars show standard deviation (SD). Figure S2B. Protease activity under different glycerol concentrations. Relative protease activity compared to $20 \%$ glycerol standard. Error bars indicate SD. Figure S3A. Inhibition of protease activity by different aprotinin concentrations. Protease activity in the presence of control inhibitor; aprotinin was shown as percentage of enzyme only control. Concentrations $\leq 10 \mu \mathrm{M}$ aprotinin significantly differ from the blank (buffer only) at $100 \mu \mathrm{g} / \mathrm{mL}$ recombinant protease concentration. Error bars indicate SD. Figure S3B. A fixed amount of DENV protease was subjected to various concentrations of an inhibitor. Aprotinin, showing trend over time. All sample reaction mixtures contain $100 \mu \mathrm{g} / \mathrm{mL}$ NS2B-NS3pro and $100 \mu \mathrm{M}$ Bz-nKRR-MCA suspended in $200 \mathrm{mM}$ Tris-HCl with $20 \%$ glycerol, pH 9.5, unless otherwise specified. Error bars indicate standard deviation. (DOCX $1640 \mathrm{~kb}$ )

\section{Abbreviations}

Ac, acetyl; AMC/MCA, 7-amino-4-methylcoumarin; Bz, benzoyl; DENV, dengue virus; DMSO, dimethyl sulfoxide; HCV, hepatitis C virus; HIV, human immunodeficiency virus; HRP, horseradish peroxidase; $I_{50}$, half maximal inhibitory concentration; IMAC, immobilized metal affinity chromatography; $K_{m}$, Michaelis constant; MNTD, maximum nontoxic dose; MTT, 3-(4,5dimethylthiazol-2-yl)-2,5-diphenyltetrazolium bromide; nKRR, norleucine-lysinearginine-arginine; NS, nonstructural protein/s; OPD, o-phenylenediamine dihydrochloride; PCR, polymerase chain reaction; PVDF, polyvinylidene fluoride; SDS-PAGE, sodium dodecyl sulfate polyacrylamide gel electrophoresis

\section{Acknowledgements}

We would like to thank Astellas Pharma Inc. for providing the compounds, Takako Nishi, Rohitha Muthugula, Bui Thu Thuy, and Muhareva Raekiansyah from the Department of Virology, Institute of Tropical Medicine, Nagasaki University for technical support during experimentation, as well as Richard M. Kinney (Center for Disease Control and Prevention, Division of Vector-borne Diseases, Fort Collins, CO, USA) for providing the infectious cDNA clone of the DENV2 16681 strain

This study was conducted (in part) at the Joint Usage/Research Center on Tropical Disease, Institute of Tropical Medicine, Nagasaki University, Japan.

\section{Funding}

This work was supported by a Health and Labor Sciences Research Grant on Emerging and Re-emerging Infectious Diseases from the Japanese Ministry of Health, Labor and Welfare, Health and Labor Sciences Research Grants (Grants in aid). A sponsorship grant from Astellas Pharma Inc. was also received in support of this research.

The Japanese Ministry of Health, Labor and Welfare had no role in the design, testing, analysis, and writing of the report. Astellas Pharma played no role in the analysis, interpretation, and writing of the manuscript.

\section{Availability of data and materials}

Supporting data were attached as an additional file.

\section{Authors' contributions}

KM, GELU, and KO conceived and designed the study. GELU and KO performed the experiments. GELU and KM analyzed the data. GELU, KM, and $\mathrm{KO}$ wrote the paper. All authors read and approved the final manuscript.

\section{Competing interests}

The authors declare that they have no competing interests.

\section{Consent for publication}

Not applicable.

\section{Ethics approval and consent to participate}

Not applicable.

\section{Author details}

'Department of Virology, Institute of Tropical Medicine, Nagasaki University, 1-12-4 Sakamoto, Nagasaki 852-8523, Japan. ${ }^{2}$ Graduate School of Biomedical Sciences, Nagasaki University, Nagasaki, Japan. ${ }^{3}$ Leading Graduate School

Program, Nagasaki University, Nagasaki, Japan.

Received: 19 May 2016 Accepted: 18 July 2016

Published online: 10 August 2016

\section{References}

1. Belaganahalli MN, Maan S, Maan NS, et al. Genetic characterization of the tick-borne orbiviruses. Viruses. 2015;7:2185-209.

2. Edillo FE, Sarcos JR, Sayson SL. Natural vertical transmission of dengue viruses in Aedes aegypti in selected sites in Cebu City, Philippines. J Vector Ecol. 2015;40:282-91.

3. Buckner EA, Alto BW, Lounibos LP. Vertical transmission of Key West dengue-1 virus by Aedes aegypti and Aedes albopictus (Diptera: Culicidae) mosquitoes from Florida. J Med Entomol. 2013;50:1291-7.

4. Marchand $\mathrm{E}$, Prat $\mathrm{C}$, Jeannin $\mathrm{C}$, et al. Autochthonous case of dengue in France, October 2013. Euro Surveill. 2013;18:20661.

5. Kutsuna S, Kato Y, Moi ML, et al. Autochthonous denque fever, Tokyo, Japan, 2014. Emerg Infect Dis. 2015;21:517-20. 
6. Nedjadi T, El-Kafrawy S, Sohrab SS, Despres P, Damanhouri G, Azhar E. Tackling dengue fever: current status and challenges. Virol J. 2015;12:212.

7. Rudolph KE, Lessler J, Moloney RM, Kmush B, Cummings DAT. Incubation periods of mosquito-borne viral infections: a systematic review. Am J Trop Med Hyg. 2014;90:882-91.

8. Martina BE, Koraka P, Osterhaus AD. Dengue virus pathogenesis: an integrated view. Clin Microbiol Rev. 2009;22:564-81.

9. Halstead SB. Controversies in dengue pathogenesis. Paediatr Int Child Health. 2012;32 Suppl 1:5-9.

10. Capeding MR, Tran NH, Hadinegoro SR, et al. Clinical efficacy and safety of a novel tetravalent dengue vaccine in healthy children in Asia: a phase 3, randomised, observer-masked, placebo-controlled trial. Lancet. 2014;384: 1358-65.

11. Hadinegoro SR, Arredondo-Garcia JL, Capeding MR, et al. Efficacy and longterm safety of a dengue vaccine in regions of endemic disease. $\mathrm{N}$ Engl J Med. 2015;373:1195-206

12. Coudeville $\mathrm{L}$, Baurin $\mathrm{N}$ and Vergu E. Estimation of parameters related to vaccine efficacy and dengue transmission from two large phase III studies. Vaccine. 2015. http://dx.doi.org/10.1016/j.vaccine.2015.11.023. Accessed 21 Nov 2015, ISSN 0264-410X.

13. Nitsche C, Holloway S, Schirmeister T, Klein CD. Biochemistry and medicinal chemistry of the dengue virus protease. Chem Rev. 2014;114:11348-81.

14. Yin Z, Patel SJ, Wang WL, et al. Peptide inhibitors of dengue virus NS3 protease. Part 1: warhead. Bioorg Med Chem Lett. 2006;16:36-9.

15. Wu C-F, Wang S-H, Sun C-M, Hu S-T, Syu Jr W. Activation of dengue protease autocleavage at the NS2B-NS3 junction by recombinant NS3 and GST-NS2B fusion proteins. J Virol Methods. 2003;114:45-54.

16. Kinney RM, Butrapet $S$, Chang GJ, et al. Construction of infectious CDNA clones for dengue 2 virus: strain 16681 and its attenuated vaccine derivative, strain PDK-53. Virology. 1997;230:300-8.

17. Tomlinson SM, Watowich SJ. Anthracene-based inhibitors of dengue virus NS2B-NS3 protease. Antiviral Res. 2011;89:127-35.

18. Junaid M, Chalayut C, Sehgelmeble Torrejon A, et al. Enzymatic analysis of recombinant Japanese encephalitis virus NS2B(H)-NS3pro protease with fluorogenic model peptide substrates. PLoS One. 2012;7, e36872.

19. Junaid M, Angsuthanasombat C, Wikberg JE, Ali N, Katzenmeier G. A straightforward experimental approach to expression, purification, refolding, and enzymatic analysis of recombinant dengue virus NS2B(H)-NS3pro protease. Biochemistry (Mosc). 2013;78:920-4.

20. Tomlinson SM, Watowich SJ. Substrate inhibition kinetic model for West Nile virus NS2B-NS3 protease. Biochemistry. 2008;47:11763-70.

21. Steuer C, Heinonen $\mathrm{KH}$, Kattner L, Klein CD. Optimization of assay conditions for dengue virus protease: effect of various polyols and nonionic detergents. J Biomol Screen. 2009;14:1102-8.

22. Kinoshita $H$, Mathenge EG, Hung NT, et al. Isolation and characterization of two phenotypically distinct dengue type-2 virus isolates from the same dengue hemorrhagic Fever patient. Jpn J Infect Dis. 2009;62:343-50.

23. Voller A, Bartlett A, Bidwell DE, Clark MF, Adams AN. The detection of viruses by enzyme-linked immunosorbent assay (ELISA). J Gen Virol. 1976;33:165-7.

24. Neubig RR, Spedding M, Kenakin T, Christopoulos A. International Union of Pharmacology Committee on Receptor N and Drug C. International Union of Pharmacology Committee on Receptor Nomenclature and Drug Classification. XXXVIII. Update on terms and symbols in quantitative pharmacology. Pharmacol Rev. 2003;55:597-606.

25. Sebaugh JL. Guidelines for accurate EC50/IC50 estimation. Pharm Stat. 2011 10:128-34

26. Li J, Lim SP, Beer D, et al. Functional profiling of recombinant NS3 proteases from all four serotypes of dengue virus using tetrapeptide and octapeptide substrate libraries. J Biol Chem. 2005;280:28766-74.

27. lempridee $T$, Thongphung $R$, Angsuthanasombat C, Katzenmeier G. A comparative biochemical analysis of the NS2B(H)-NS3pro protease complex from four dengue virus serotypes. Biochim Biophys Acta Gen Subj. 2008;1780:989-94.

28. Niyomrattanakit $P$, Winoyanuwattikun $P$, Chanprapaph $S$, Angsuthanasombat C, Panyim S, Katzenmeier G. Identification of residues in the dengue virus type 2 NS2B cofactor that are critical for NS3 protease activation. J Virol. 2004;78:13708-16.

29. Leung $D$, Schroder $K$, White $H$, et al. Activity of recombinant dengue 2 virus NS3 protease in the presence of a truncated NS2B co-factor, small peptide substrates, and inhibitors. J Biol Chem. 2001;276:45762-71.

30. Phong WY, Moreland NJ, Lim SP, Wen D, Paradkar PN, Vasudevan SG. Dengue protease activity: the structural integrity and interaction of NS2B with NS3 protease and its potential as a drug target. Biosci Rep. 2011;31: 399-409.

31. Niyomrattanakit P, Yahorava S, Mutule I, et al. Probing the substrate specificity of the dengue virus type 2 NS3 serine protease by using internally quenched fluorescent peptides. Biochem J. 2006;397:203-11.

32. Schuller A, Yin Z, Brian Chia CS, et al. Tripeptide inhibitors of dengue and West Nile virus NS2B-NS3 protease. Antiviral Res. 2011;92:96-101.

33. Ghosh AK, Osswald HL, Prato G. Recent progress in the development of HIV-1 protease inhibitors for the treatment of HIV/AIDS. J Med Chem. 2016.

34. Arakaki TL, Fang NX, Fairlie DP, Young PR, Martin JL. Catalytically active dengue virus NS3 protease forms aggregates that are separable by size exclusion chromatography. Protein Expr Purif. 2002;25:241-7.

35. Viswanathan U, Tomlinson SM, Fonner JM, Mock SA, Watowich SJ. Identification of a novel inhibitor of dengue virus protease through use of a virtual screening drug discovery Web portal. J Chem Inf Model. 2014:54: 2816-25.

36. Jadhav A, Ferreira RS, Klumpp C, et al. Quantitative analyses of aggregation, autofluorescence, and reactivity artifacts in a screen for inhibitors of a thiol protease. J Med Chem. 2010;53:37-51.

37. Thorne N, Auld DS, Inglese J. Apparent activity in high-throughput screening: origins of compound-dependent assay interference. Curr Opin Chem Biol. 2010;14:315-24.

38. Turek-Etienne TC, Small EC, Soh SC, et al. Evaluation of fluorescent compound interference in 4 fluorescence polarization assays: 2 kinases, 1 protease, and 1 phosphatase. J Biomol Screen. 2003;8:176-84.

39. Simeonov A, Jadhav A, Thomas CJ, et al. Fluorescence spectroscopic profiling of compound libraries. J Med Chem. 2008;51:2363-71.

40. Shannon AE, Chappell KJ, Stoermer MJ, et al. Simultaneous uncoupled expression and purification of the dengue virus NS3 protease and NS2B COfactor domain. Protein Expr Purif. 2016;119:124-9.

41. Kim YM, Gayen S, Kang C, et al. NMR analysis of a novel enzymatically active unlinked dengue NS2B-NS3 protease complex. J Biol Chem. 2013;288: 12891-900.

\section{Submit your next manuscript to BioMed Central and we will help you at every step:}

- We accept pre-submission inquiries

- Our selector tool helps you to find the most relevant journal

- We provide round the clock customer support

- Convenient online submission

- Thorough peer review

- Inclusion in PubMed and all major indexing services

- Maximum visibility for your research

Submit your manuscript at www.biomedcentral.com/submit
) Biomed Central 